\title{
Image Compression using Decision Tree Based SVD-ASWDR Technique
}

\author{
Kanchan Bala ${ }^{1}$ and Deepinder Kaur ${ }^{2}$ \\ ${ }^{1}$ Research scholar (Computer science and research dept.) \\ Punjab Technical University, Jalandhar \\ ${ }^{2}$ Assistant professor (Computer science and research dept.) \\ Punjab Technical University, Jalandhar \\ ${ }^{1}$ kanchukashyap@gmail.com \\ 22deepinderkaur.bhullar@gmail.com
}

\begin{abstract}
The image compression is the technique in which image is compressed to reduce its size and complexity. The two type of compressions are used which are lossy and lossless compressions. The lossy is the type of compression which information of the image is lost at the time of compression. The ASWDR algorithm is the lossy type of compression algorithm which access textural and color features of the image. To improve efficiency of ASWDR algorithm decision tree is used for classification. The decision tree classifies the pixels which are required and which are not required and not required are removed from the image. The proposed technique performs well in terns of PSNR, MSE and compression ratio.
\end{abstract}

Keywords: Classification, WDR, ASWDR, Compression ratio, PSNR, MSE

\section{Introduction}

\subsection{Image Compression}

As the multimedia based [1] web applications are increased so there is an increase in the required bandwidth \& storage capacity. The solution to the problem is image compression. Image compression is a procedure by which the size of the image is reduced without degrading the image quality so that more images are stored in a given memory space. Image compression is of two types lossless \& lossy. In case of lossless the compressed image is exactly same as original image. It minimizes the bit rate without distortion of the image. Lossy image compression is used where some details of image can be lost to save bandwidth or storage space.

\subsection{Various Image Compression Techniques}

Different algorithm for compression are DCT,DWT, Vector, quantization, SVD etc.SVD is a method of image compression to discard the singular value with low amount of image info[2]. Here the image data is expressed in form of number of Eigen values to perform compression. To meet low complexity $8 \mathrm{Pt}$ DCT is used [3]. Here the DCT approximation is based on polar decomposition methods the approximation transform is done to meet low complexity requirements as compared to dct and sdct hence reduces power consumption. In this $8 \mathrm{pt}$ det technique a modification to standard det matrix by means of rounding off operation is done. This technique is useful for compression of images with high compression ratio. Another technique is the DWT [4] where the size of original image is decreased by estimating DWT coefficients below a certain value. It captures both location \& frequency information. This technique first decomposes an 
image into coefficients called sub bands $\&$ then these resulting coefficients are compared with threshold \& the coefficients below threshold are set to $0 \&$ rest are encoded using lossless image compression technique. It provides sufficiently high compression ratio and maintains image quality. In dwt image is first divided into a set of high pass (detail) \& low pass (approximate) coefficients. The image is first divided into blocks of $32 * 32$. Each block is then passed through the filters: the first level decomposition is then performed to decompose the input data into an approximation \&detail coefficients. After obtaining the transformed matrix, the detail \& approximate coefficients are separated as LL, LH, HL \&HH coefficients. All coefficients are discarded except the LL coefficients that are transformed into second level. The coefficients are then passed through a constant scaling factor to achieve the desired compression ratio. Another technique DCT image compression [5] is used for converting a signal into elementary frequency components. It attempts to de correlate the image data. After de correlation each transform coefficient can be encoded independently without losing compression efficiency.

\section{Literature Review}

Y.Wongsawat, h.ochoa, k.r Rao (2004): In this paper, modified hybrid DCT-SVD image encoding system is used to encode both monochromatic and color images. The SVD requires high complexity to compute Eigen values and vectors. DCT performs well for highly correlated image. Hence both are combined to obtain a hybrid DCT-SVD image coding algorithm (HDCTSVD). DCT is used when the standard deviation of an $8 * 8$ sub block of image is below threshold else SVD [16] is used. K.H Talukder and K. Harada (2007): In this paper they have discussed Haar wavelet approach for image compression on a low complex 2D image \& quality assessment of the image is done after compressions. In the 2D image compression using Haar wavelet [17] from a given matrix a transformed matrix is obtained by following operations of averaging and differencing. Prabhakar. Telagarapu, V.Jagan Naveen (2011): In this paper they have discussed DCT and wavelet transform for image compression is. DCT [18] attempts to de correlate image data. In the proposed DCT algorithm the image is broken into $8 * 8$ blocks of pixels. From left to right and top to bottom \& then DCT is applied to each block. Each block is then compressed through quantization. When desired image is reconstructed through decompression, which uses inverse discrete cosine transform (IDCT). M.M.H. Chowdhury and A Khatun (2012): In this paper they have discussed about the discrete wavelet technique of image compression. They have also compared the performance of DWT with other techniques. Proposed technique is based on the discrete wavelet transformation [19] that gives less complexity and also maintains image quality. Ramandeep Kaur \& Navneet Randhawa (2012): In this paper they have discussed the hybrid technique of DCT \& DWT [20] for image compression. It is specifically used for the compression of images where there is tolerable degradation allowed. The algorithm used performs the discrete cosine transform (DCT) on the discrete wavelet transform (DWT) coefficients and then discards majority of high coefficients to obtain compression. S.Kahu \& R.rahate (2013): In this paper they have discussed image compression technique svd [21]. It is based on the factor $\mathrm{k}$ which represents the number of Eigen values. The factor $\mathrm{K}$ impacts the quality of image. If $\mathrm{k}$ is equal to the rank of matrix then amount of compression is very less \& quality is nearly same as original image. As $\mathrm{k}$ increases image quality improves but more storage space is required. In case the value of $\mathrm{k}$ is smaller the compression ratio is more but image quality deteriorates. Therefore it is necessary to choose value of $\mathrm{k}$ depending on requirement of storage or image quality. K. saraswathy, D vaithiyanthan \& R. sesha sayanan (2013): In this paper they have discussed about the orthogonal approximation of the 8 pt DCT [22]. DCT approximation is based on polar decomposition methods. The approximation transform is done to meet low complexity requirements as compared to dct and sdct hence reduces power 
consumption. In dct we leave the unwanted frequency components while retaining required ones. Alok Kumar Singh \& G.S Tripathi (2014): In this paper they have done a comparative study of DCT, DWT \&Hybrid(DCT-DWT)[23] .Transform on the basis of parameters compression ratio(CR), mean square root(MSE), peak to signal ratio (PSNR).The individual DCT \& DWT techniques are also discussed along with the procedure of hybrid technique.

\section{WDR Algorithm for Image Compression}

Two different wavelet based techniques are WDR \& ASWDR. Wavelet transforms are widely studied over the last decade [7-8]. WDR is a lossy technique. It is a method of efficient embedded image coding. WDR retains all the important features like low complexity region of interest, embeddedness \& progressive SNR.ASWDR adapts scanning procedure used by WDR in order to predict locations of significant transform values at $1 / 2$ thresholds. Some of the compression techniques were used earlier [8].In WDR [9] technique firstly a wavelet transform is applied to the image. After this the bit plane based WDR encoding algorithm for the wavelet coefficients is carried out. WDR [10] mainly consists of 5 steps as follows. The first step is initialization in which an assignment of a scan order is first made where a scan order is a one to one \& onto mapping $\mathrm{f} . .=\mathrm{x} \mathrm{k}$, for $\mathrm{k}=1,2,3 \ldots \mathrm{p}$, between the wavelet coefficient ()$\&$ a linear ordering $(\mathrm{x}$ k).This scan order is a zigzag through sub bands from higher to lower levels. For coefficients in sub bands row based scanning, column based scanning is used in the horizontal sub bands \& vertical sub bands respectively. Zigzag scanning is used for diagonal \& low pass sub bands. As the scanning order is made, an initial Threshold $\mathrm{T}_{\mathrm{v}}$ is set so that all transformation satisfy IX $1<\mathrm{T}_{\mathrm{v}} \&$ at least one transform value satisfies IX1> $=T_{v} / 2$.In the second step update threshold by $T \mathrm{k}=\mathrm{T} \mathrm{k} 1 / 2$. In the third step of significance pass the transform values are deemed significant if they are greater than or equal to the threshold value. Then their index values are encoded using WDR method [11].The difference reduction method essentially consists of binary encoding of the number of steps to go from the index of the last significant value to the index of the current significant value. The output from the significance pass includes the signs of significant values along with sequence of bits generated by difference reduction which describes the precise locations of significant values. Last step is the refinement pass. It is to generate the refined bits via the standard bit- plane quantization procedure. Each refined value is better approximation of an exact transform value. Repeat second to last step until big budget is reached.

\section{ASWDR Algorithm for Image Compression}

ASWDR is one of the leading enhanced image compression algorithm proposed by Walker $(2000 ; 2001)$. This technique does focus to get better the subjective qualities of compressed images and improve the results of image quality and compression ratio. ASWDR algorithm is a simple adaptation of the Wavelet Difference Reduction (WDR) algorithm. WDR algorithm uses a hard and fast ordering of the locations of wavelet coefficients, so ASWDR method uses a various order which does focus to adapt itself to specific image qualities. The ASWDR adjusts the scanning order therefore as to predict positions of recent significant values. ASWDR dynamically adapts to the locations of edge details in an image by using specific scanning order, and this increases the declaration of these edges in ASWDR compressed images. So, ASWDR shows better qualities, especially at low bit rates, than WDR. ASWDR is simply a modification of WDR to achieve higher image quality and compression rate and it leads to better results than WDR. The PSNR values from reconstructed images by using ASWDR compression are higher than WDR compression. MSE values from reconstructed image by ASWDR 
compression are lower than WDR compression. The ASWDR technique performs better than the WDR compression technique.

\section{Proposed Methodology}

In proposed technique the image is firstly compressed by SVD-ASWDR technique and then wavelet transform is applied on it. After extracting features with wavelet transform the patches are created and patches are sorted in order to perform compression by using decision tree. Decision tree sort the patches according to NRL order that means it define root node which maximum weight, left node which has less weight than root node and right node which has minimum weight. In this way the patches are sorted in descending order in terms of its weight (information). Now we can see the leaf nodes have the least amount of information (weight). In order to achieve compression of the image the leaf nodes which have least amount of information are discarded to reconstruct the image. Then inverse wavelet transform is applied to decompress the image. When the ASWDR technique is applied decision tree classifier the features which are not required are removed from the image in the efficient manner and increase compression ratio.

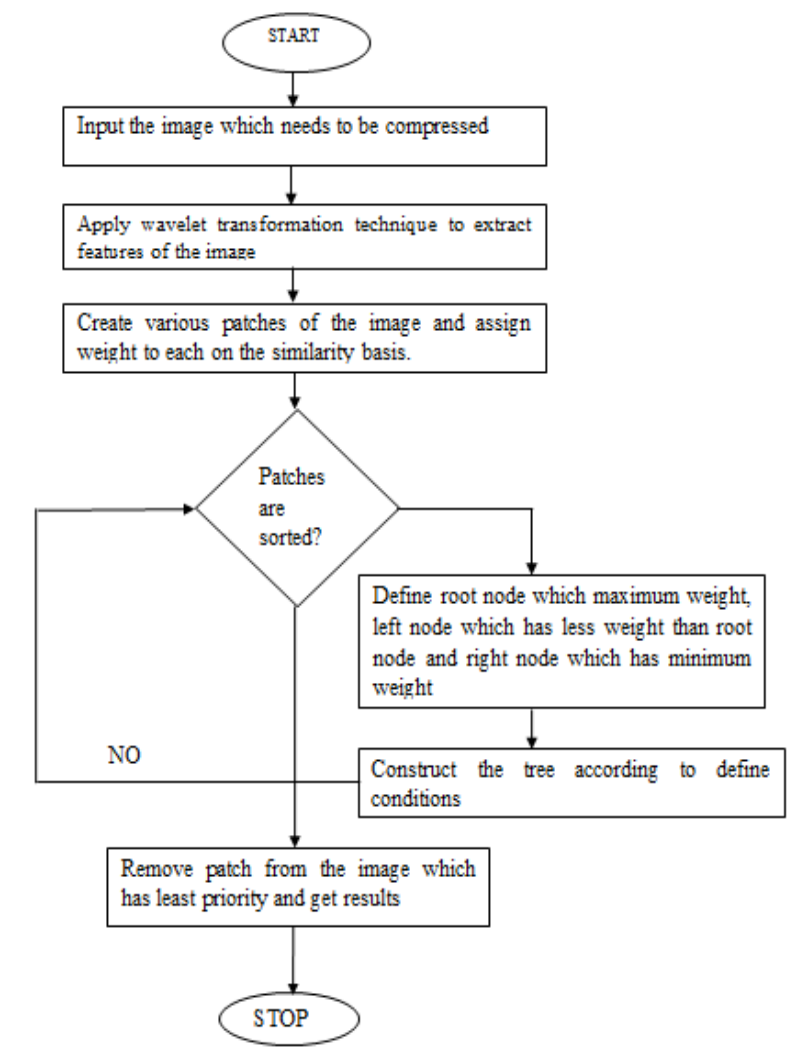

\section{Experimental Results}

The proposed and existing algorithms are implemented in MATLAB. The MATLAB is the tool which is used to perform complex mathematical computations and used to analyze results visually and graphically.

\subsection{Results of SVD-ASWDR}

In this figure, image 'chess.tif' is compressed at various compression ratios. This figure shows that the image is reconstructed at 55 coefficients. 


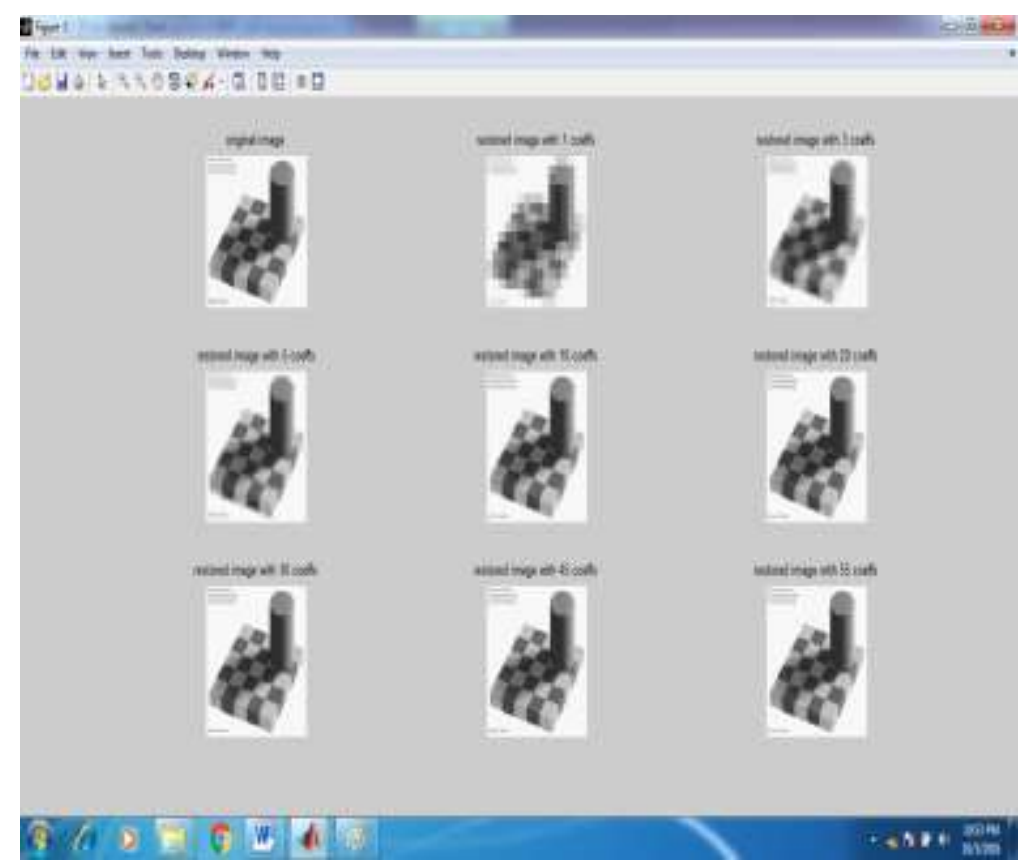

Figure 1. Reconstructed Image 'chess.tif'

This graph shows that we have reconstructed the image by using 55 coefficients under the SVD-ASWDR technique. The $\mathrm{x}$ - axis represents the number of coefficients taken for reconstruction and $y$-axis represents the PSNR values with respect to compression rate.

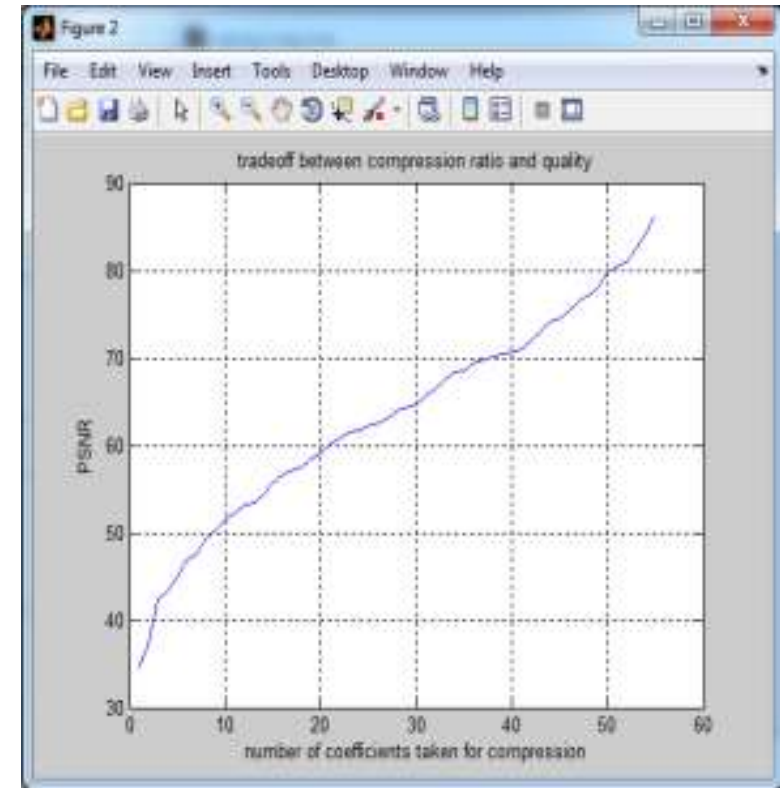

Figure 2. Performance of SVD-ASWDR

\subsection{Results of Decision Tree Based SVD-ASWDR}

In Figure 3, image is compressed by using enhanced SVD-ASWDR technique. In this SVD ASWDR is enhanced by using decision tree. 


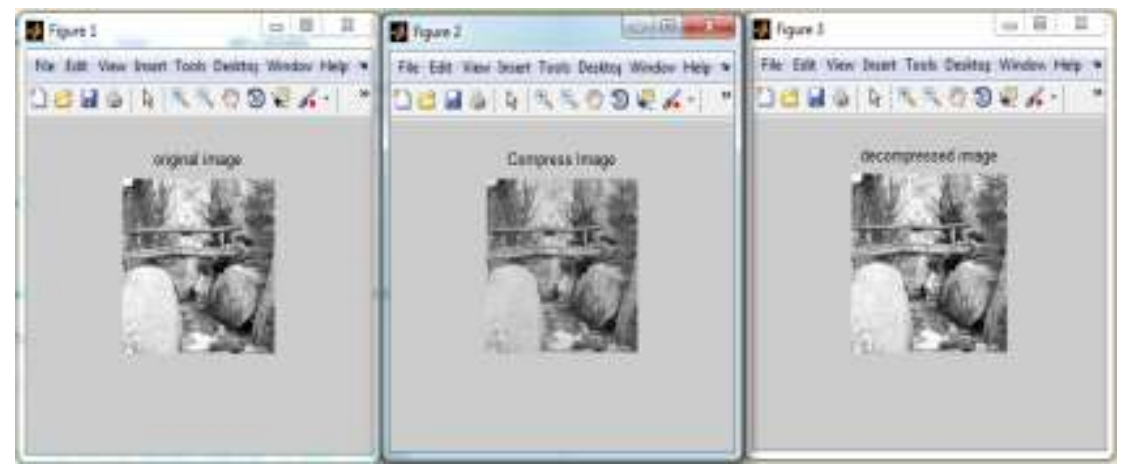

Figure 3. Implementation of Enhanced SVD-ASWDR

\subsection{Comparison}

Table 1, shows that the results obtained by enhanced SVD-ASWDR which is based on decision tree, outperforms the results of SVD-ASWDR that is existing technique.

Table 1. Comparison of SVD-ASWDR and enhanced SVD-ASWDR

\begin{tabular}{|c|l|c|c|}
\hline Parameters & Image/method & SVD-ASWDR & Decision tree based SVD-ASWDR \\
\hline PSNR & Bridge.tif & 27.12 & 30.21 \\
CR & & 0.577 & 0.609 \\
\hline PSNR & Chess.tif & 25.99 & 31.12 \\
CR & & 0.586 & 0.612 \\
\hline PSNR & Roots.tif & 22.82 & 29.22 \\
CR & & 0.571 & 0.596 \\
\hline PSNR & Football.tif & 26.96 & 30.96 \\
CR & & 0.511 & 0.630 \\
\hline PSNR & Cameraman.tif & 22.98 & 30.78 \\
CR & & 0.564 & 0.619 \\
\hline PSNR & X-ray.tif & 30.75 & 31.96 \\
CR & & 0.612 & 0.620 \\
\hline PSNR & Watch.tif & 27.89 & 30.44 \\
CR & & 0.497 & 0.584 \\
\hline PSNR & Storm.tif & 23.92 & 28.43 \\
CR & & 0.590 & 0.610 \\
\hline PSNR & Lena.tif & 27.91 & 30.91 \\
CR & & 0.567 & 0.604 \\
\hline PSNR & Jockey.tif & 28.93 & 31.11 \\
CR & & 0.581 & 0.611 \\
\hline Average PSNR & Total Images (10) & 27.75 & 32.23 \\
Average CR & & 0.516 & 0.616 \\
\hline
\end{tabular}

The graph represented in Figure 4, and Figure 5, shows that the results of PSNR and compression ratio (CR) obtained by Decision Tree based SVD-ASWDR outperforms the SVD-ASWDR technique.

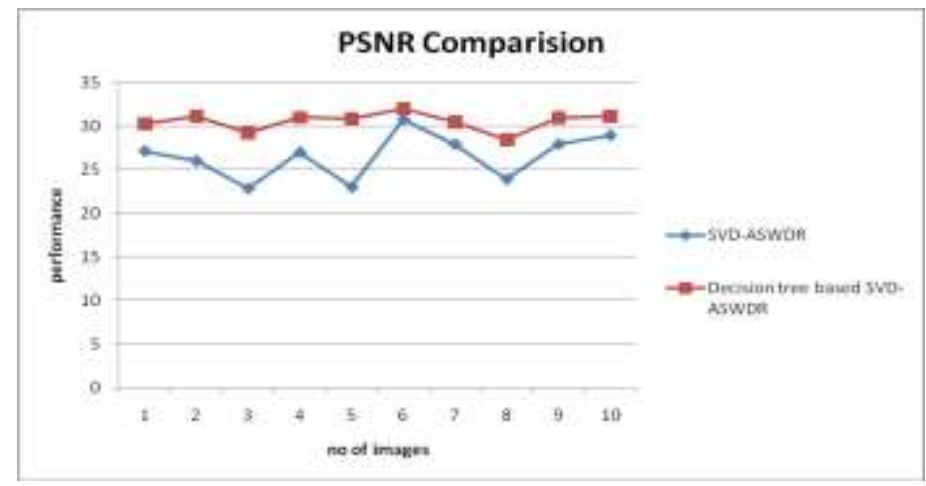

Figure 4. Graphical Comparison in Terms of PSNR 


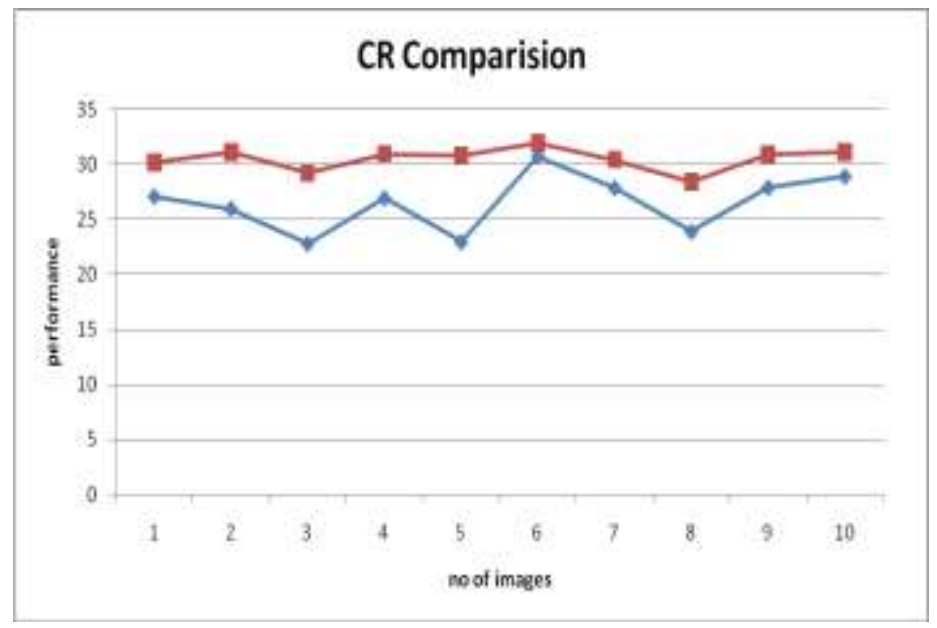

Figure 5. Graphical Comparison in Terms of PSNR

\section{Conclusion}

In this work, it is been concluded that ASWDR is the technique of lossy image compression. The ASWDR technique is based on textual and color features of the image. The decision classifier is used to classify the required and non-required features of the image. To reduce complexity of the algorithm decision classifier can be replaced with decision tree classifier. The proposed and existing techniques are implemented in MATLAB and proposed technique performs well in terms of PSNR, MSE and compression ratio.

\section{References}

[1] S. Jayaraman, E. Rajan and T. Veerakumar, "Digital Image Processing, PSG College of technology, Coimbatore", Tamil Nadu.

[2] S. Kahu, and R. Rahate, "Image compression using singular value decomposition", International Journal of Advancements in Research \& Technology, vol. 2, no. 8, (2013), pp. 244-248.

[3] K. Saraswathy, D. Vaithiyanathan and R. Seshasayanan, "A DCT approximation with low complexity for image compression", International Conference on Communications and Signal Processing (ICCSP), Melmaruvathur, (2013), pp. 465-468

[4] M. M. H. Chowdhury and A Khatun, "Image compression using discrete wavelet transform", International Journal of Computer Science vol. 9, no. I, (2012), pp.327-330.

[5] P. Telagarapu, Y. J. Naveen, A. L. Prasanthi and G. Y. Santhi, "Image compression using OCT and wavelet transformations", Int. J. of Signal Processing, Image Processing and Pattern Recognition, vol. 4, no. 3, (2011).

[6] "Images of Science: Science of Images," organized by Albert V. Crewe, held, , at the National Academy of Sciences, (1992) January 13-14.

[7] M, Antonini, M. Barlaud, P. Matieu and I. Daubechie, "Image coding using wavelet transforms".

[8] G. Strang and T. Nguyen, "Wavelet \& filter Banks", Wellesley- Cambridge Press, Boston, (1996).

[9] J. Tian and R. O. Wells." Alossy image codec based in index coding", IEEE Data compression conference, (1996) December 96.

[10] J. S. Walker and T. O. Nguyen." Adaptive scanning methods for wavelet difference reduction in lossy image compression”, Proceedings of IEEE International Conference on Image Processing, 2000

[11] Neghahdaripur and A. Khamene, "Motion- based compression of underwater video imagery for operations of unmanned summersible vehicles".

[12] Mallat, "A wallet Tour of signal processing", Academic Press., (1998).

[13] A. Said and W. A. Pearlman, "Anew, fast, and efficient image codec based on set partitioning in hierarchal trees".

[14] A. Said and W. A. Pearlman, "Anew, fast, \& efficient image codec based on set partitioning in hierarichal trees", IEEE on circuits \& systems for video Technology, vol. 6, no. 3, (1996).

[15] G. M. Davis and A. Nosratinia. "Wavelet - based - image coding: An overview applied \& computational control”, Signals \& circuits, vol. 1, no. 1, (1998).

[16] Y. Wongsawat, H. Ochoa, K. R. Rao and S. Oraintara, "A modified hybrid DCT-SVD image-coding system for color image". 
[17] K. H. Talukder and K. Harada, "Haar wavelet based approach for image compression and quality assessment of compressed image".

[18] P. Telagarapu, Y. J. Naveen, A. L. Prasanthi and G. Y. Santhi, "Image compression using OCT and wavelet transformations".

[19] M. M. H. Chowdhury and A. Khatun, "Image compression using discrete wavelet transforms".

[20] Er. R. K. Grewal and N. Randhawa, "Image compression using discrete cosine transform \& discrete wavelet transform".

[21] S. Kahu and R. Rahate, "Image compression using singular value decomposition".

[22] K. Saraswathy, D. Vaithiyanathan and R. Seshasayanan. "A DCT approximation with low complexity for image compression".

[23] A. K. Singh and G. S. Tripathi, "A comparative study of OCT, DWT \& hybrid (DCT-DWT) transform". 